\title{
How does physical activity benefit people living with dementia? A systematic review to identify the potential mechanisms of action
}

\author{
Jan Pringle, Ruth Jepson, Alison Dawson, Louise McCabe and Alison Bowes
}

\begin{abstract}
Purpose - One limitation of research that assesses the effectiveness of physical activity interventions for people with dementia is that most do not describe the intervention in sufficient detail to ascertain a theoretical basis or mechanism of action that determines the effective components. This paper aims to identify studies which evaluate the mechanisms of action of physical activity interventions for people with dementia, to further inform effective intervention development.

Design/methodology/approach - Papers were screened for evidence of evaluation of specific forms of physical activity, using pre-defined inclusion criteria. Analysis was conducted to ascertain if mechanisms of action were corroborated by data within and between studies.

Findings - The authors identified 26 studies with a measured mechanism of action; these related to the effects of physical activity on either neurological structure or endocrinal markers, including hormones. Physical activity had potential to reduce hippocampal atrophy, increase neural recruitment, activate the noradrenergic system and improve anti-inflammatory responses. While individual studies were hampered by small sample sizes, the body of evidence indicated that physical activity may have potential to delay cognitive decline.

Practical implications - Mechanisms of action in relation to dementia and physical activity are likely to be multifaceted, and physical activity may be protective against progression in the early stages of cognitive decline. Physical activity may be of greatest benefit if incorporated into on-going lifestyle, rather than engaged in for short periods, and combined with social interaction.
\end{abstract}

Originality/value - This paper is unique in its focus on the mechanisms of action of physical activity interventions for people with dementia.

Keywords Systematic Review, Ageing, Physical activity, Dementia, Alzheimer's,

Cognitive impairment or decline

Paper type Literature review

\section{Background}

Dementia, as a condition, is of global concern, with around 50 million people worldwide living with the disorder, and an estimated $10 \%-20 \%$ of cases being preventable $(\mathrm{WHO}$, 2019). Physical activity is amongst the healthy lifestyle factors that may reduce the likelihood of developing dementia, or slow the progress of cognitive decline (Lewis et al., 2020; WHO, 2019)

While the general benefits of physical activity are well documented (Reiner et al., 2013), it has been estimated that around a quarter of adults are insufficiently active (WHO, 2019). Environmental factors, such as living in a safe neighbourhood with open spaces, can positively influence activity levels amongst healthy adults [National Institute for Heath and
Jan Pringle and

Ruth Jepson both are based at the Scottish Collaboration for Public Health Research and Policy, Edinburgh, UK. Alison Dawson, Louise McCabe and Alison Bowes are based at the Faculty of Social Sciences, University of Stirling, Stirling, UK.

Received 30 September 2020 Revised 5 November 2020 Accepted 12 November 2020

(C) Jan Pringle, Ruth Jepson Alison Dawson, Louise McCabe and Alison Bowes. Published by Emerald Publishing Limited. This article is published under the Creative Commons Attribution (CC BY 4.0) licence. Anyone may reproduce, distribute, translate and create derivative works of this article (for both commercial and non-commercial purposes), subject to full attribution to the original publication and authors. The full terms of this licence may be seen at http://creativecommons.org/ licences/by/4.0/legalcode

Funding: Healthcare Management Trust. 
Care Excellence (NICE), 2018]; however, for those with physical or mental impairments, keeping active may be more challenging (Bartlo and Klein, 2011). For example, people with conditions such as dementia may face particular difficulty due to the effects of the condition on memory, thought processes, behaviour and ability to perform everyday activities (Alzheimer's Society, 2015). Despite these challenges, studies of physical activity programmes for older adults suggest improved cognition (Colcombe and Kramer, 2003), increased independence (Mechling, 2008), and improved functional ability (Bowes et al., 2013). If physical activity is undertaken in a group it can also increase social connection and reduce feelings of loneliness and isolation (Kim et al., 2014).

Understanding the underlying or causal pathways that explain how such interventions produce their outcomes is an essential step towards informing further implementation (Moore et al., 2014; gov.uk, 2018). One limitation of research that assesses the effectiveness of physical activity and behavioural change interventions is that most do not describe the intervention in sufficient detail to ascertain whether there is a theoretical basis, or underlying mechanism of action, to determine the effective (or ineffective) components (Bowes et al., 2013). It is therefore difficult to replicate effective interventions, or identify the factors that contribute to effectiveness across interventions. Without such detail, researchers and practitioners aiming to develop and evaluate complex interventions may be unable to proceed with confidence (Bowes et al., 2013).

A mechanism of action describes the manner in which a beneficial (therapeutic) agent or activity works, including the functional (biological, physiological or biochemical) processes within the body that produce a given response (Medical Dictionary, 2019). These responses have traditionally referred to medication (pharmacological) treatments. However, over the last couple of decades there has been increasing recognition that non-pharmaceutical interventions, in conjunction with improved understanding of the underlying condition, may produce better benefits; even in 2004 Douglas et al. emphasised that pharmacological treatments for dementia should be used as a second line approach and that nonpharmacological options should, in best practice, be pursued first.

We therefore sought to identify studies that might provide detail relating to mechanisms of action as the active or "operative" elements of physical activity interventions for people with dementia, via a systematic review. The focus was on human studies, rather than evidence that might have been derived from animal studies, due to our interest in informing practical interventions for people with dementia. Improved understanding of mechanisms of action can help to inform theories of change, and therefore more effective intervention development.

\section{Methods}

\section{Approach}

A systematic review was carried out to assess current evidence relating to the effects of physical activity for people with dementia, and identify studies detailing specific and welldefined mechanisms of actions.

\section{Search terms and databases}

Searches combined key words related to dementia with terms for exercise and physical activity, as used in our previously reported scoping study of physical activity for people with dementia (Bowes et al., 2013). Key words and an example search string are shown in Table 1. The review sought to better understand mechanisms of action of interventions which require physical exertion. The language used to report and describe such interventions can vary between cultures and academic disciplines, for which reason, whilst acknowledging the distinction between the two, search terms were included to capture both "exercise" and "physical activity" and variants thereof. 
Including terms around specific physical activities potentially allows consideration of mechanism of action at different levels of physical exertion.

The dementia-related search terms were purposely designed to capture studies of interventions aimed at people living with a range of dementias; the earlier scoping review found that studies used participant eligibility criteria ranging from diagnosis of specific dementias (e.g. Alzheimer's disease) to observed cognitive impairment suspected to be due to dementia. It is acknowledged that in practice there may be the co-existence of symptoms of multiple or mixed dementias at, or following, a dementia diagnosis. However, given the potential wide variation of symptoms and abilities, even between people with the same diagnosis or measured stage of their condition, it was felt important to examine and report on people with a broad range of cognitive impairment.

Individual searches were conducted using each exercise or activity-related term, with intradatabase duplicates being removed. All study designs could be included, subject to meeting other inclusion criteria. An approach to study inclusion which does not privilege specific study designs can provide insight into the shape and volume of the "research landscape" in fields of enquiry where, due to ethical or practical challenges, fewer randomised or clinical controlled trials may take place. The approach has previously been employed successfully in reviews of a range of other topics (Bowes et al., 2016; Dawson et al., 2015; Rutherford et al., 2019). The study design of included studies is identified and discussed in the findings section of this paper.

Publication dates up until August 2020 were included; databases accessed: Web of Knowledge (includes BIOSYS and Medline databases), CINAHL, ASSIA, Social Services Abstracts, Embase, PsycINFO, and the British Nursing Index.

\section{Study selection}

Papers were included if they described or evaluated a specific form of physical activity (rather than referring to physical activity in general), and identified a specific primary research study. In relation to outcomes, we aimed to identify if a mechanism of action had been suggested, and measured, in each study, therefore building on the evidence synthesis of exercise and dementia conducted by Lewis et al. (2020).

Inclusion and exclusion criteria for the review are summarised in Table 2.

With further regard to the inclusion/exclusion criteria, the term 'adults' referred to anyone over 18 years of age; no other lower age limit was set, to avoid excluding people with early onset conditions.

We were unable to include dissertations or theses, due to the typical length of such pieces of work. However, where shorter summaries of such work had been published, these were included, if the published work fulfilled all criteria for the review.

\section{Applying the inclusion criteria}

Results of individual searches were combined to produce a single dataset for each database. Within and between database duplicates were removed, titles and abstracts screened for relevance, followed by full text screening of remaining papers. Two members

\section{Table 1 Search terms and example search string}

dement*Alzheimer*(Lewy* exercis*(physical activit*)swim*gym*walk* danc*yoga((tai TS=((dement* OR Alzheimer* OR "Lewy* bod*" bod* $^{*}($ cognit* impair*) chi) or (tai ji))stretch*sport*(physical train*) OR "cognit* impair*") AND exercis*) 
Study design: specific primary research studies of any design, which include an intervention and outcome(s)English languagePopulation: adults (18yrs or older) with dementia or mild cognitive impairment, living independently or in a care environment; human studiesIntervention: defined, specific physical activity interventionsOutcomes: physiological, functional, psychological, or social outcomes that are attributable to physical activity interventionA stated and measured mechanism of action
Study design: study protocols without outcomes; commentary papers; dissertationsFull article not available or not in English languagePopulation: no dementia; congenital or traumatic cognitive impairment (e.g. brain injury); animal studies; populations under 18yrs of agelntervention: no specific physical activity intervention; physical activity incidental to other factors/actions; risk or prevalence studies (as sole focus)Outcomes: no specific, separate, or identifiable effects of physical activity (e.g. multimodal studies where outcomes relating specifically to effect of physical activity are not reported) of the review team blind screened papers during this process, with a third member of the review team acting as an arbitrator in cases of disagreement.

\section{Data extraction and synthesis}

Key information for studies was extracted into an evidence table, designed to capture essential data relating to participants, type of dementia, type, duration and frequency of physical activity, outcome measures, and suggested mechanism of action. Studies were examined to ascertain if the mechanism of action was corroborated by evidence, and findings/conclusions summarised (see Table 3 in results section).

\section{Quality appraisal}

Studies were appraised in relation to method, participant characteristics, selection bias, statistical power, outcome measures and validity of findings, using a mixed method appraisal tool (Pluye et al., 2011).

\section{Findings}

The Preferred Reporting Items for Systematic Reviews and Meta-Analyses (PRISMA) flow chart (Figure 1) is used to summarise the literature review process and results (Moher et al., 2009).

Following title and abstract, then full paper screening, 142 papers were found that discussed a potential mechanism of action; we further sought to identify whether the mechanism was directly measured. For example, a study might suggest that physical activity may improve neural plasticity without measuring any evidence of effect within the brain. Alternatively, another study might suggest improvements in brain neurotropic factors as a result of an exercise programme, and measure this through blood samples.

We identified 26 studies with a specified and measured mechanism of action. Details of these studies are given in Table 3.

\section{General overview}

The included studies were conducted in a wide range of countries: USA (8), Canada (3), Taiwan (3), Brazil (2), Iran (2), South Korea (2), Czech Republic (1), Denmark (1), Hong Kong (1), Pakistan (1), Portugal (1), Saudi Arabia (1). This indicates the global nature of interest, and the range of populations/ethnic groups that have been involved in research on this topic. 


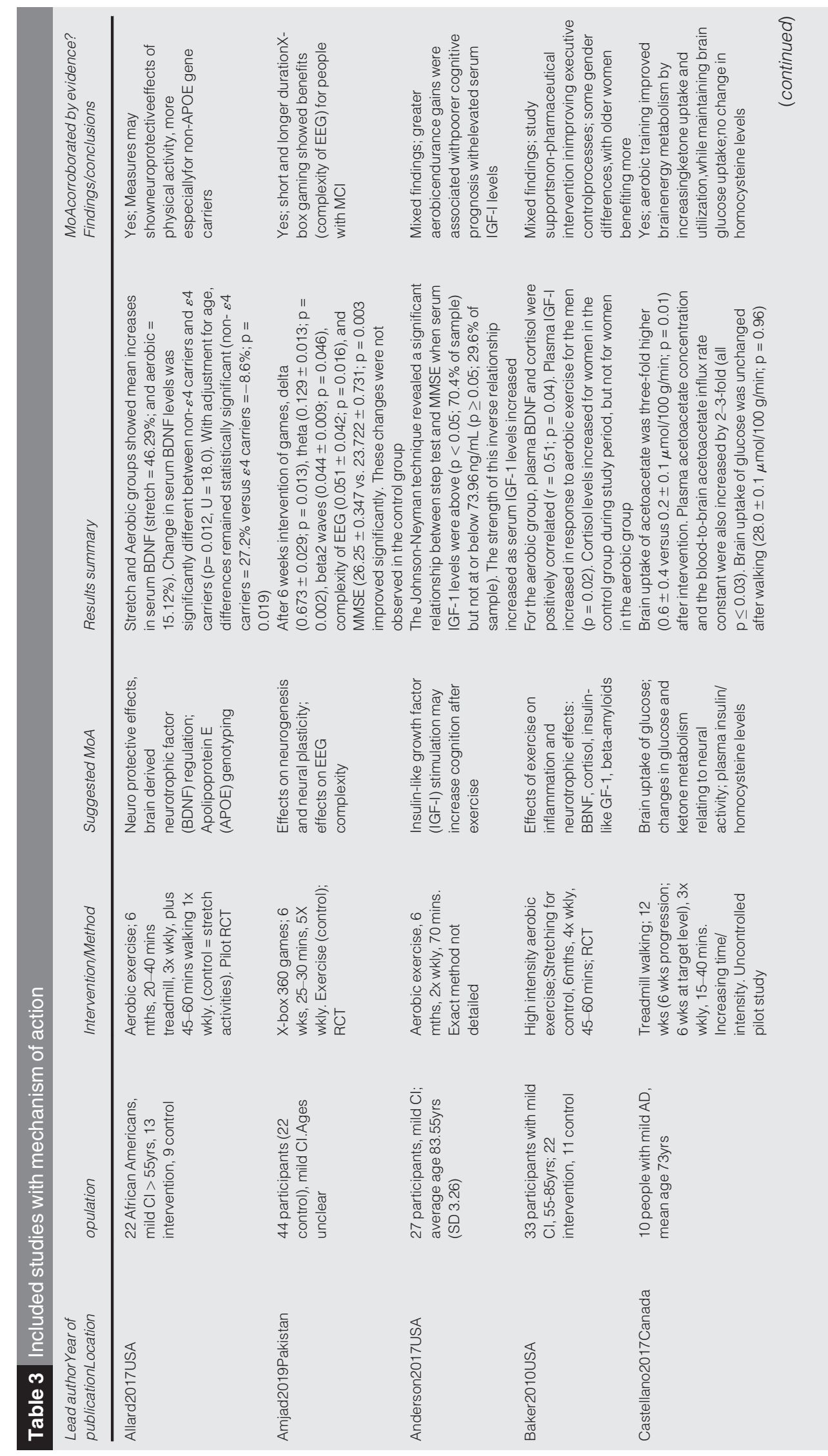




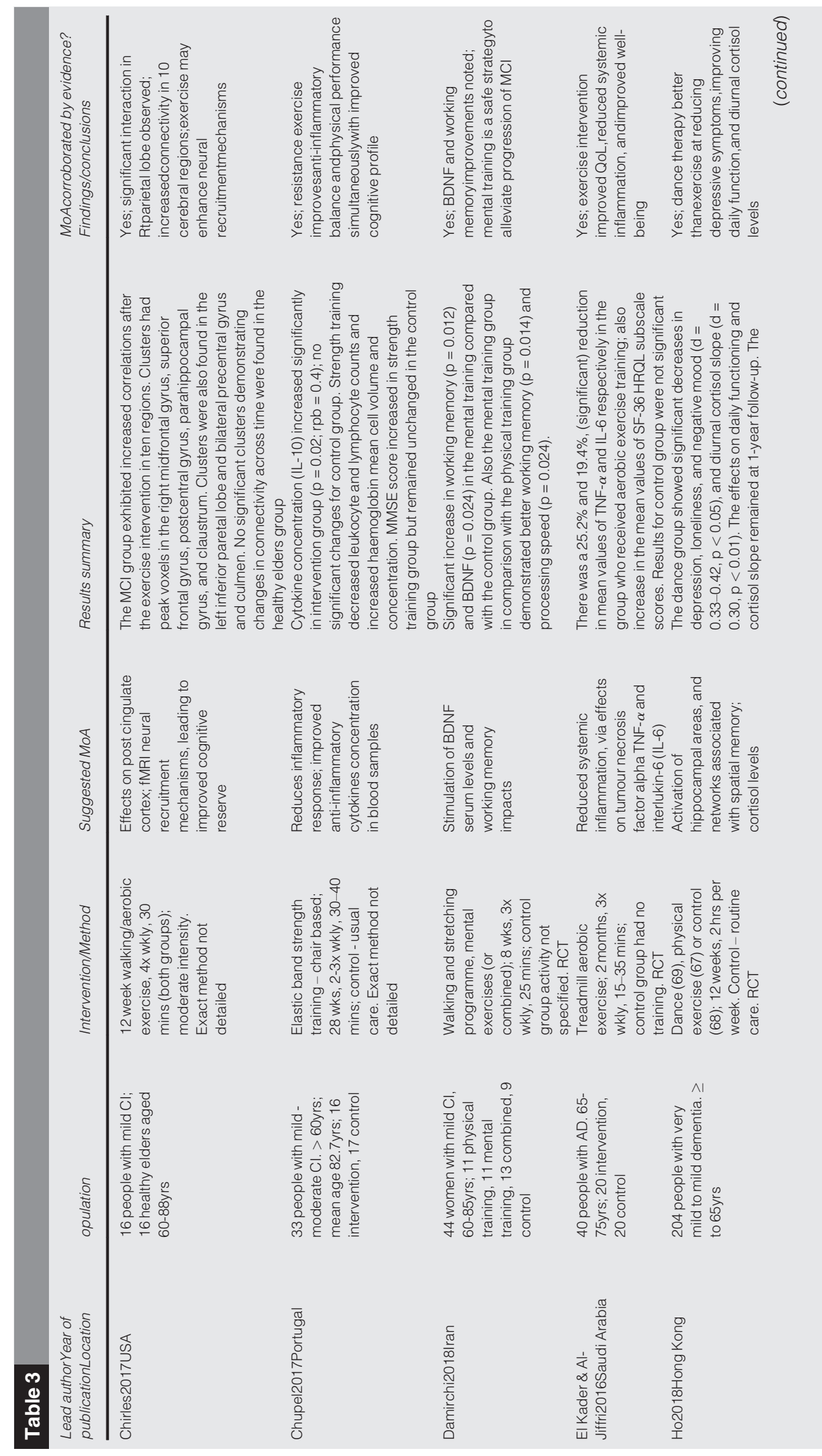




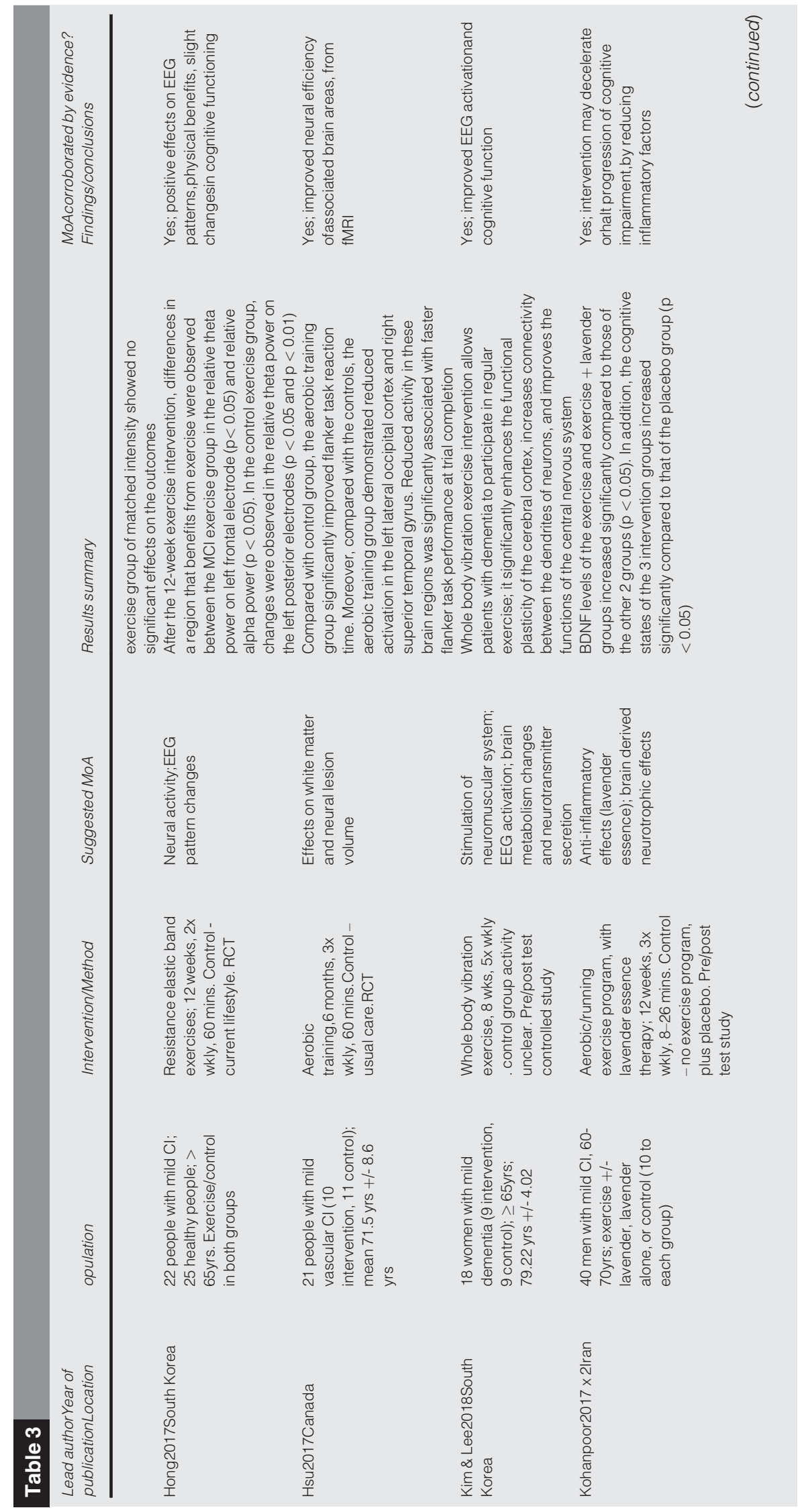




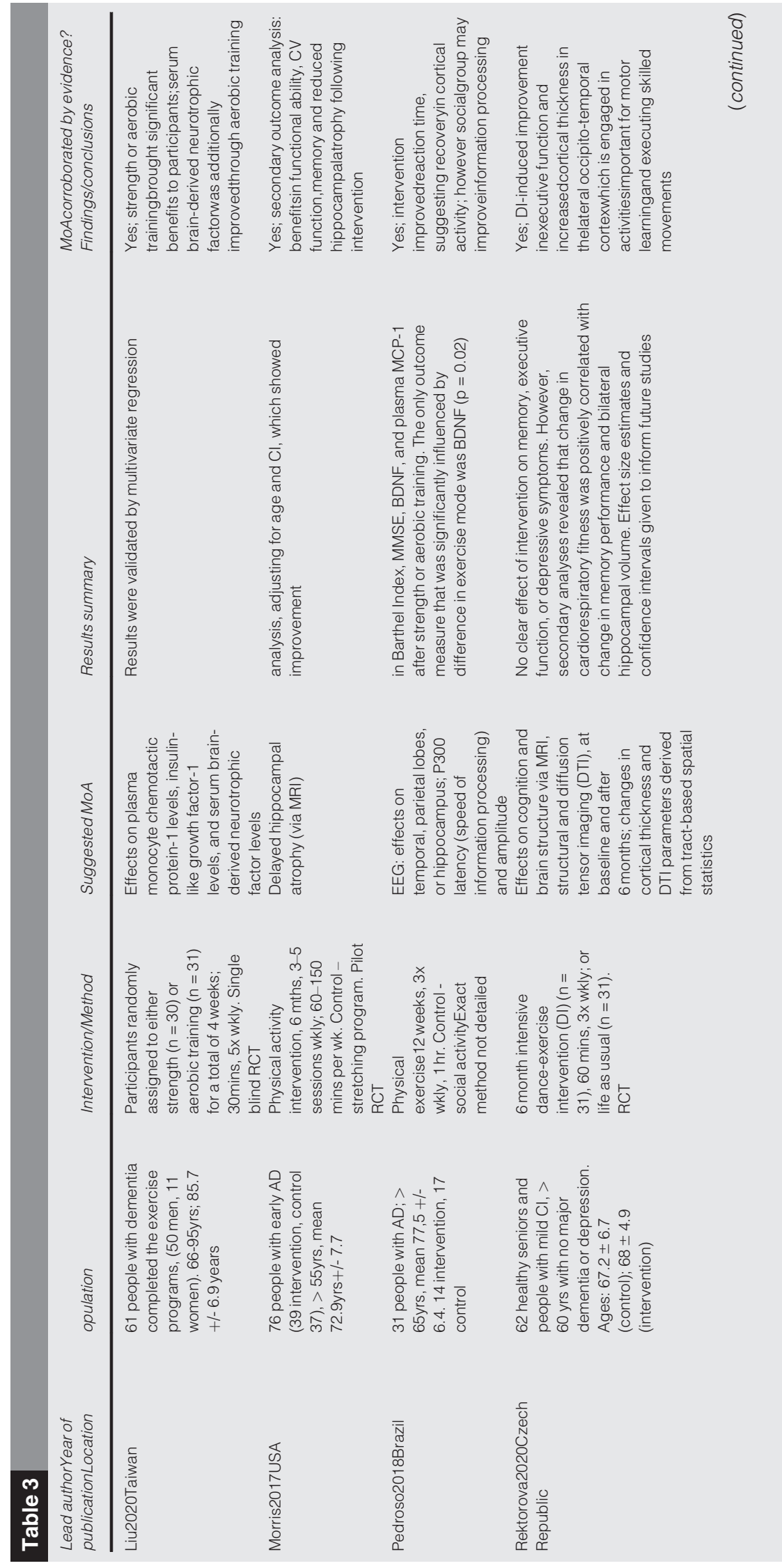




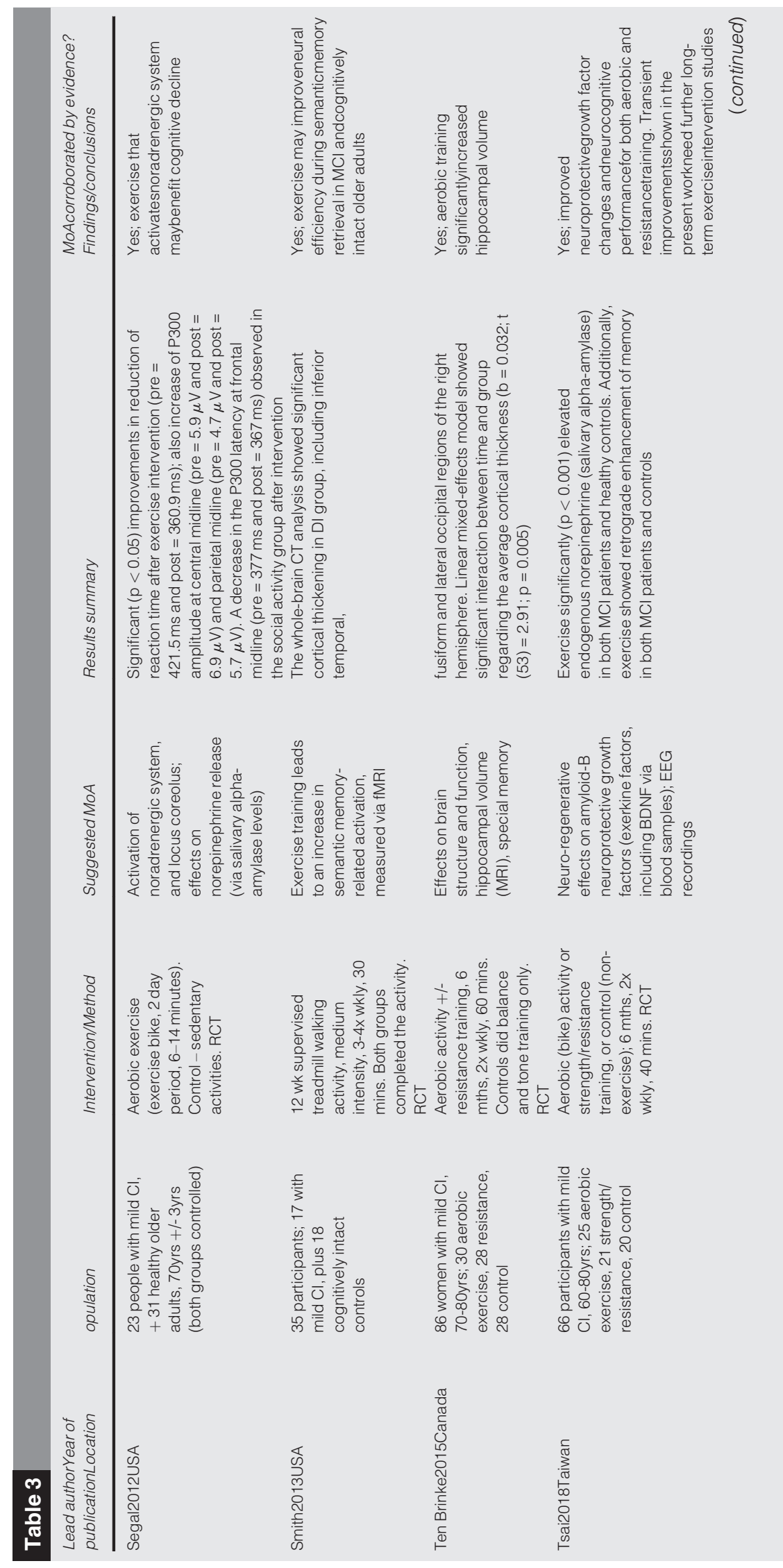


$>\frac{1}{0}$

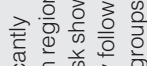

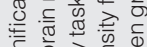

कक

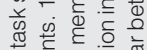

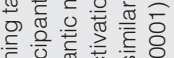

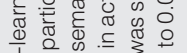

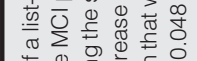

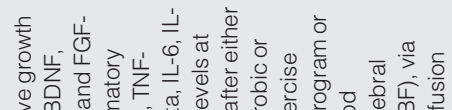

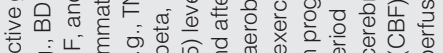

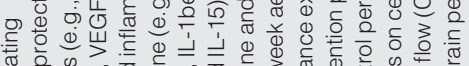

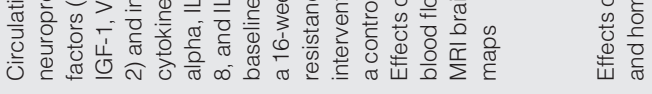

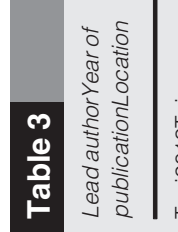

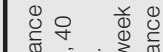

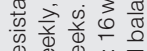

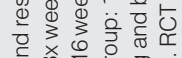

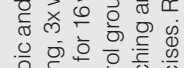

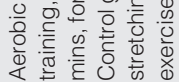

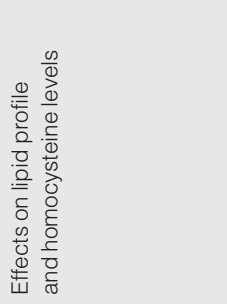

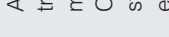
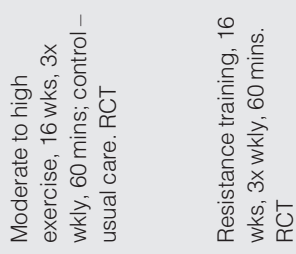

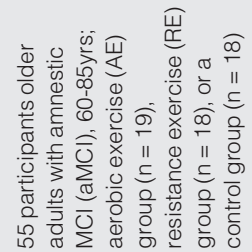

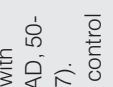
定

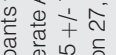

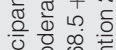

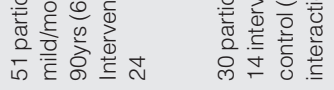

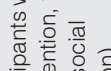

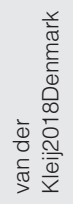

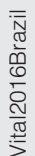




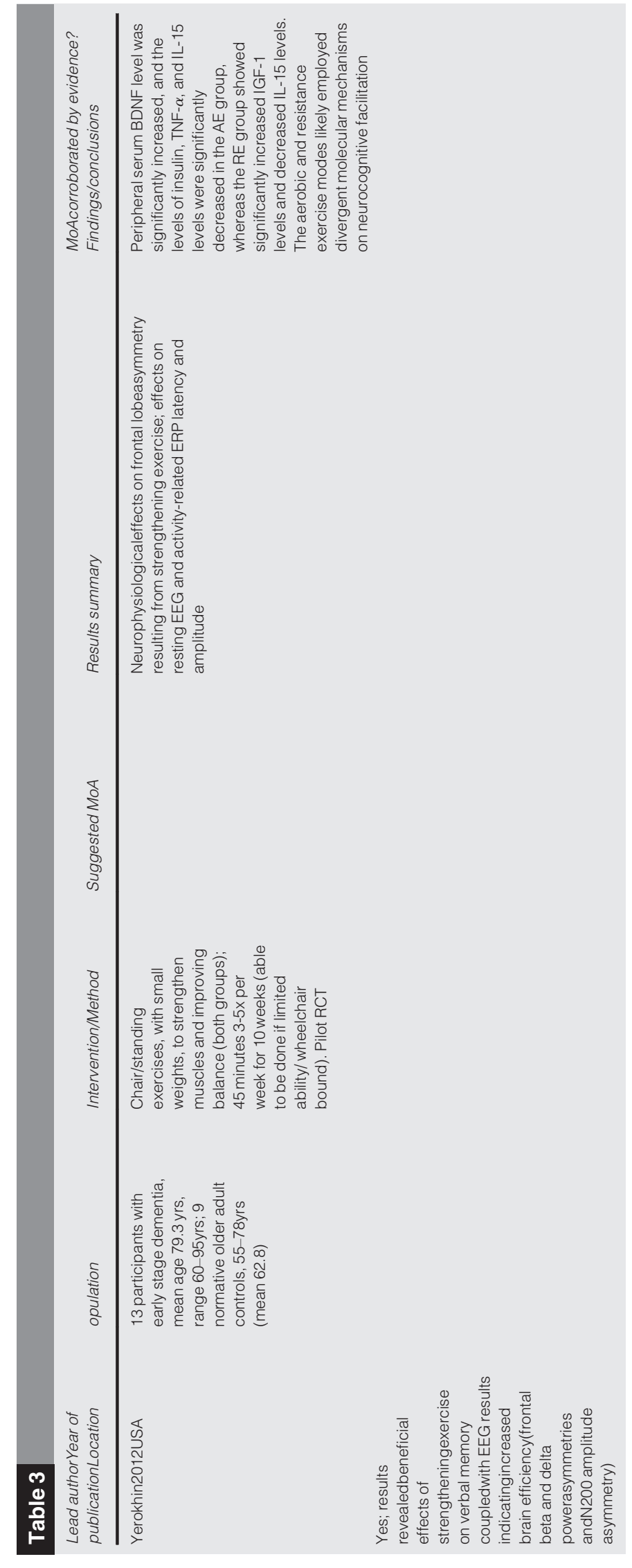

VOL. 22 NO. 12021 QUALITY IN AGEING AND OLDER ADULTS | PAGE 13 


\section{Figure 1 PRISMA Flow Diagram}

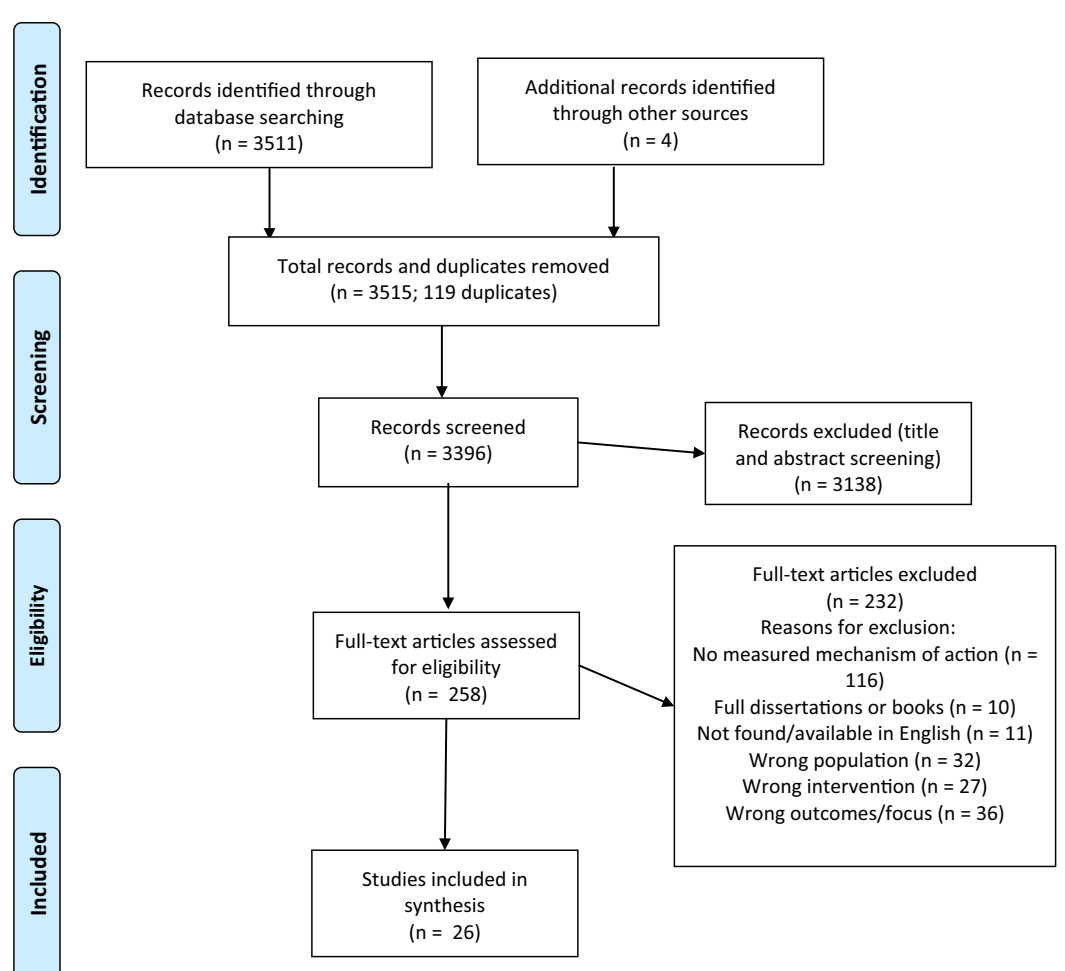

Of the 26 studies, 16 were randomised controlled studies, three pilot RCTs, one other uncontrolled pilot study, and two pre/post-test studies; 4 studies did not detail an exact study method. All studies had been published within the past 10 years, with around $75 \%$ dating from 2017 onwards. This suggests a more recent focus and interest in the determination of mechanisms of action, and the operational elements of interventions.

Study participant numbers were generally small, with only eight studies recruiting over 50 participants (see Table 3 for further details). This perhaps reflects difficulties in conducting studies with, and recruiting, vulnerable groups of people (Economic and Social Research Council, 2019). With regard to diagnosis or degree of cognitive impairment, the majority of studies $(n=18)$ included participants with mild cognitive impairment. Six studies had participants with a diagnosis of Alzheimer's disease (mild to moderate), and two studies recruited participants with mild dementia.

\section{Quality appraisal}

Quality appraisal was hampered by lack of comparability of studies, and low statistical power due to small study numbers. In addition, differences such as disease stage and progression, intervention intensity and duration, and gender, all impacted on the ability to make direct comparisons between studies. However, inclusion criteria were well described in all studies, and outcome measures relevant; due to the nature of research and the participants, controlled trials were generally single-blind. Baseline characteristics between intervention and control groups were well described, but not comparable due to cognitive impairment being absent in some control subjects. 


\section{Mechanisms of action}

In the included papers, measured mechanisms of action fell into two broad categories, determining:

1. structural or functional changes, measured either through brain imaging (e.g. MRI, fMRI) or EEG readings; and

2. levels of substances such as endocrines and hormones in blood or saliva samples

Structural and functional changes in the brain were reported in 11 studies, and the remaining studies reported on physiological mechanisms. Findings from these two broad categories, and their sub categories, are examined below.

While the focus was mainly on measuring elements within these two broad categories, there was only minor acknowledgement of the social benefits of taking part in a group activity, and any behavioural change mechanisms of action that may have resulted from the interventions. We were therefore unable to report in any depth on these elements, although a few pertinent points are made, as relevant.

\section{Structural or functional measures}

\section{Hippocampal volume}

Of the studies measuring structural changes, 3 examined hippocampal volume, using brain magnetic resonance imaging (MRI) techniques (Morris et al., 2017; Ten Brinke et al., 2015; Van der Kleij et al., 2018). Morris reported benefits in functional ability, improved memory and reduced hippocampal atrophy (compared to control) following a 6 month physical activity programme for people with early Alzheimer's disease. Ten Brinke et al. (2015) reported significantly increased hippocampal volume (as opposed to reduced atrophy) following aerobic activity for a similar period of time. However, Van der Kleij et al (2018) found no consistent effects from a 16 week programme, and questioned whether this period of intervention time was sufficient to show a difference in a relatively small sample of 51 participants. Sample sizes in the other two studies were not much bigger, but Van der Kleij et al. did include participants with more moderate cognitive impairment, whereas participants in the other two studies had either mild or early changes only. All three studies had similar intervention types (aerobic activity of moderately to high intensity, 2-3 times a week) over a 4-6 month period.

\section{Neural recruitment and activation}

Studies by Chirles et al. (2017), Smith et al. (2013) and Hsu et al. (2017) used functional magnetic resonance imaging (fMRI) to measure changes in brain activation, with all 3 studies reporting improvements following their aerobic activity programmes for participants with mild cognitive impairment. Increased activity in 10 cerebral regions was reported by Chirles et al. (2017). Similar improvements in associated brain areas were reported by Hsu et al. (2017); in the study by Smith et al. (2013), a list-learning task significantly improved, and 11 brain regions showed increased activation during the memory task. Chirles et al. (2017) concluded that exercise may enhance neural recruitment mechanisms, and lead to improved cognitive reserve. Activity programmes lasted 3-6months, were aerobic in nature, and sample sizes were small, with 10-17 intervention participants in these studies (see Table 3 for individual study details).

\section{Neural connectivity}

Neural connectivity effects were measured using electro-encephalogram (EEG) readings in 5 studies (Amjad et al., 2019; Hong et al., 2017; Kim and Lee, 2018; Pedroso et al., 2018; 
Yerokhin et al., 2012). All studies reported improved EEG patterns/complexity, indicating improved neural connectivity; cognitive function was improved too, although improvements were only slight in the case of Hong et al. (2017). Again, intervention numbers were small (9-22). Intervention durations were shorter than other studies, ranging from 6-12 weeks, with less emphasis on intensity (e.g. X-box training (Amjad et al., 2019), or whole body vibration (Kim and Lee, 2018).

In the study by Pedroso et al. (2018), while the physical activity intervention improved reaction time, the control group took part in a social activity, which also resulted in improved information processing. This was the only study that had a defined/explicit social activity for control participants, although other group activities (intervention or control) may have had an implicit social element.

\section{Endocrine and hormonal measures Cytokines}

Several studies used measures of endocrine activity; these included cytokines (see definitions at the end of the paper) such as C-reactive protein, tumour necrosis factor-alpha, beta amyloid and interlukin-6. Cytokines are a large group of proteins, peptides or glycoproteins that are secreted by specific cells of the immune system; they regulate inflammation and haematopoiesis (formation of red blood cells). All these biomarkers can be associated with cognitive decline pathology in diseases such as Alzheimer's (Baker et al., 2010). Studies that measured cytokines included Baker et al. (2010), Chupel et al. (2017), El-Kader and Al-Jiffri (2016), Liu et al. (2020), and Tsai et al., 2019) all of which reported improved anti-inflammatory and cognitive results in the exercise intervention groups, although study numbers were small (intervention groups $\leq 31$ ). However, exercise was seen as having potential as a beneficial non-pharmaceutical intervention for people with mild to moderate cognitive impairment.

In associated study measures, brain derived neurotrophic factor (BDNF) was also examined in seven studies (Allard et al., 2017; Baker et al., 2010; Damirchi et al., 2018; Kohanpour et al. (2017); Liu et al., 2020; Tsai et al., 2018, 2019). BDNF is the most prevalent growth factor in the central nervous system (CNS). It is essential for the development of the CNS, new cell production, and for neuronal plasticity (Autry and Monteggia, 2012). Benefits of exercise were shown (via BDNF levels) to be greater for differing groups in these studies: for example, the study by Allard et al. (2017) concluded that neuroprotective effects of physical activity were greater in those who were non-APOE gene carriers, whereas Baker et al. (2010) concluded that older women may benefit to a greater extent, according to BDNF levels. In comparison, the study by Damirchi et al. (2018) found that mental training or combined mental/physical training outperformed (via BDNF levels) physical training alone for older women with mild cognitive impairment; intensity and duration (8weeks) in this latter study were lower than other studies examining similar interventions (for example, the Allard and Baker studies were both of 6 months duration). A 12 week intervention of exercise \pm lavender extract exposure in the study by Kohanpour et al. (2017) showed increased BDNF levels and improved cognitive states in a sample of people with mild cognitive decline. There were no other immediately discernable differences in sample populations (e.g. ages, baseline cognitive levels etc.) to explain the differences found by these studies.

In terms of exercise type, all interventions in this subgroup examined aerobic-type activities; Tsai et al. $(2018,2019)$ compared aerobic and resistance-style exercise, and found both to be of benefit over 16-24 week periods, but concluded that longer term interventions are required to determine if benefits from either exercise type are sustained. The results from Liu et al. (2020) indicated that both strength and aerobic training programs over the course of 4 weeks can bring about significant benefits for patients with dementia in both their ADLs 
and cognitive performance. Furthermore, the difference in results between the two modes of exercise was not significant.

\section{Hormonal measures}

Other markers of interest in the included studies included hormonal measures of Insulin-like growth factor (IGF-I), cortisol, and norepinephrine.

Insulin-like growth factor (IGF-I) is a peptide hormone that functions primarily to stimulate growth but that also possesses some ability to decrease blood glucose levels, having insulin-like actions in some tissues, though far less potent than insulin in decreasing blood glucose concentrations [Britannica Dictionary (science division), 2019]. IGF-I levels were measured in studies by Anderson et al. (2017), Baker et al. (2010), Liu et al. (2020), and Tsai et al. $(2018,2019)$. In these studies, results were mixed, with Tsai and colleagues reporting some acute exercise increases in IGF-I levels, but indicating that these increases might not be sustained following completion of the activity. Baker et al. (2010) found increases in IGF-I levels for men, but overall better cognitive outcomes for women with mild cognitive impairment. Liu et al (2020) reported that neither strength nor aerobic exercise had a significant effect on IGF-1 levels. In the study by Anderson et al. (2017) researchers found that contrary to the study expectations, greater exercise endurance was associated with poorer cognitive prognosis when serum IGF-I levels were above a certain level $(73.96 \mathrm{ng} / \mathrm{mL})$. They concluded that the higher IGF-I levels might indicate disease progression within their sample, potentially as a compensatory response, and therefore participants with higher IGF-I levels may be less likely to benefit from the intervention.

Cortisol is a steroid hormone, often called the "stress hormone" because of its connection to the stress response (Hormone.org, 2019). Studies by Baker et al. (2010) and Ho et al. (2018) examined cortisol levels, the former as plasma cortisol, the latter via salivary samples. Baker et al. (2010) reported stable rather than increasing cortisol levels in their aerobic exercise group (both sexes), rather than the increasing levels (for women only) in their control (stretching exercise) group. In their male (stretching) control participants cortisol levels dropped.

Ho et al. (2018) reported lower cortisol levels in both intervention types (dance therapy and physical exercise compared to control), with the dance therapy group showing greater improvements (i.e. lower cortisol levels) that were sustained over 1 year follow-up. Numbers in this study were higher than other studies, with interventions being undertaken by 137 participants, who had very mild or mild dementia.

Norepinephrine (also called noradrenaline or noradrenalin), acts as a hormone and neurotransmitter [Britannica Dictionary (science division), 2019], and was measured in the study by Segal et al. (2012) via salivary alpha-amylase biomarkers. This study concluded that exercise activates the noradrenergic system, which may, in turn, benefit cognitive decline in both those with and without existing cognitive decline (intervention and control subjects). The authors of this study concluded that the benefits of exercise may work through more than one mechanism. This certainly seems to be the case across the included studies, and this will be considered further in the discussion section.

All studies measuring hormonal markers had fairly high intensity physical activities. Differences in duration type and intensity will be discussed further below.

\section{Discussion}

\section{Summary of findings and comparison with previous reviews}

This review has concentrated on primary studies that directly measure and analyse the mechanisms of action of physical activity interventions for people with cognitive decline. 
In the past, Douglas et al. (2004) stated that non-pharmacological options should be pursued as a first line of treatment for people with dementia. At that time, their review contained a small section on activity therapies (dance, sport, drama), but concluded that details outlining the mechanism of change underpinning such activity-based interventions was lacking (Douglas et al., 2004).

A later review by Haeger et al. (2019) focussed on structural and functional changes following physical activity (measured via MRI), and concluded that the effects of exercise mainly impacted on brain structures sensitive to neurodegeneration. Our current review updates and builds on Haeger et al. by also examining changes in endocrine and hormonal measures; it demonstrates that progress has been made in recent years in terms of investigating mechanisms of action, but also indicates where further knowledge is still required.

As previously mentioned, there were very few references to the potential effects of socialising within activity (or control) groups, which may have additional memory-protective effects for older adults (Pan and Chee, 2019). Behavioural change mechanisms of action that may have resulted from the interventions were also not investigated in the included studies, but may have had an impact (Junge et al., 2018). Within the overall body of evidence from the review, however, there was certainly sufficient cause for physical activity to be viewed as a potential means of delaying the progression of cognitive decline.

\section{Disease progression}

In the included papers, the level of existing cognitive impairment appeared to have an influence on the impact of interventions. For example, where the effects of interventions on cerebral blood flow were measured, participants with more advanced cognitive impairment benefited less, possibly due to existing lower blood perfusion (van der Kleij et al., 2018). In addition, people with more advanced disease may also exhibit higher levels of substances such as IGF-I before participating in an intervention (Anderson et al., 2017); this is thought to be due to IGF-I being produced in greater quantities during neurodegeneration progression, as a response to increasing IGF-I resistance (Anderson et al., 2017). As a result, since it may be impossible to detect which study participants are deteriorating, and to what extent, cerebral blood flow and IGF-I levels may not give the most accurate indication of intervention effect. It would therefore seem advisable to determine as much information relating to cognitive ability and diagnoses as possible at the start of any study, to make any observed and measured changes easier to interpret.

\section{Intervention type and duration}

Within the interventions, there was a range of duration from intense aerobic activity, several times a week (Allard et al., 2017; Baker et al., 2010; Tsai et al., 2019) to a single 2 day, short duration intervention (Segal et al., 2012). Other interventions included X-box gaming (Amjad et al., 2019), an "exergaming" intervention, which combines video gaming with physical activity while playing.

Some activities were of sufficient duration and intensity that only people who were already physically fit would have been able to participate (Ho et al., 2018). However, interventions that were of shorter duration, and less intense, did not always appear to deliver the same benefits (Damirchi et al., 2018; Vital et al., 2016). These factors may explain the variable study results, since some of the study participants may not have had the necessary capability to participate to the same level, if at all. In addition, differing dementia sub-types, or clinical presentations, may render some participants better able to exercise than others. Overall, the evidence would tend to indicate that while physical activity can be beneficial for everyone, within their capabilities, being and remaining as active as possible throughout the 
life-course is potentially a better means of delaying or averting cognitive loss than attempting to start activities once physical and mental abilities are already declining.

Activities for control participants ranged from "usual care" to balance and tone exercises (Ten Brinke et al., 2015), stretching exercises (Allard et al., 2017; Baker et al., 2010), or exercise that was of same duration and intensity, but of a differing type (Ho et al., 2018 compared dance-movement intervention, with a similar length/intensity exercise programme, and a routine care group). Rather than being sedentary, control groups were therefore often participating in another physical activity. As stated earlier, there may have been a social interaction element also involved in the intervention or control group activities, which was not acknowledged as a compounding and potentially beneficial factor.

With further regard to activity type, dance therapy was shown to outperform exercise at reducing depressive symptoms, and improving daily function (Ho et al., 2018), which may reflect the additional elements included in dance activities, such as remembering steps, social engagement, and the effects of music (Merom et al., 2016).

\section{Gender}

The studies in this review did indicate some differences in intervention effect between men and women. For example, the greater cognitive function results shown for women in the study by Baker et al. (2010) were attributed to improvements in glucose regulation, insulin sensitivity, and reduced cortisol levels. However, the women in this study were also more sedentary than the men at baseline, potentially making similar activity intervention effects more marked. While some differences in effect for each gender were noted in a few studies, there was no consistent message with regard to gender that could be drawn from the included studies.

\section{Biomarker measures}

There were a number of questions raised about biomarkers that may have impacted on findings. For example, although several studies used IGF-I levels as an outcome measure, higher IGF-I levels can indicate increasing disease progression, potentially as a compensatory response (Vardy et al., 2007), and may not therefore be an accurate means of measuring intervention impact.

Cortisol was another biomarker that raised some interesting points. In short, increased cortisol levels can compromise brain resilience to stress, and potentially increase susceptibility to neurodegeneration (Baker et al., 2010). However, the two studies that measured cortisol levels (Baker et al., 2010 and Ho et al., 2018) measured levels in different ways (the former as one day/time point plasma cortisol levels, the latter via single day salivary samples). Cortisol levels are not only subject to day-to-day variations, they are also elevated with age, a variable that is almost three times greater for women than men (Otte et al., 2005). In addition, higher cortisol levels may be present where disease progression is more rapid (Csernansky et al., 2006). These factors call into discussion the suitability of cortisol as an accurate outcome measure for ageing participants, particularly in relation to cognitive progression and gender.

With regard to BDNF, in humans it is only possible to measure circulating BDNF, which may not accurately reflect brain level changes (Tsai et al., 2018), and make comparisons with the findings from animal studies problematic.

Homocysteine levels may also vary according to a wide range of factors, including exercise intensity, food consumption, and levels of vitamin B12 or folate (Vital et al., 2016). Measuring and controlling for such factors would be very difficult, therefore the value of findings relating to homocysteine levels may be uncertain.

Choice of biomarker outcomes would therefore seem to need careful consideration. 


\section{Strengths and limitations}

This review is unique in its focus on amalgamating evidence relating the mechanisms of action of physical activity for people with cognitive decline. Due to the diversity of variables such as intervention duration/intensity, participant characteristics and selected outcome measures, direct comparisons or meta-analyses were not feasible. However, the resulting descriptive analysis does serve to combine a key body of evidence from a range of diverse populations. While the included studies were limited by their small sample sizes, the challenges of conducting research that involves participants with cognitive decline should not be underestimated, and may necessitate reduced study numbers. However, the need to further our understanding of interventions that may be of benefit is vital, albeit within the perimeters that such research is bound to operate.

As with all systematic reviews, meticulously planned and conducted search and screening processes can still fail to capture all relevant evidence.

\section{Conclusions}

Taken as an overall body of evidence, this review has indicated the value of physical activity for people with cognitive decline. However, it seems clear that the mechanisms of action are still to be fully understood, and are likely to be multifaceted. Several areas worthy of further consideration were highlighted:

Clinical implications:

- In general, physical activity should be considered as protective against progression in the early stages of cognitive decline.

- Rather than short duration interventions, physical activity may be of greatest benefit if incorporated into on-going lifestyles.

- Physical activity combined with social interaction should be considered.

- Individual ability to participate in physical activity at the level required to promote mechanisms of action needs more consideration.

Research implications:

- Attention needs to be given to:

- choice of biomarker, taking into consideration natural changes associated with disease progression;

- levels of activity prior to intervention;

- effect and type of control activity;

- type and level of cognitive impairment;

- type and level of intervention activity;

- social mechanisms of action that may lead to benefits of taking part in activities; and

- behavioural change mechanisms of action that may have resulted from the intervention.

\section{Definitions}

Cytokines are a large group of proteins, peptides or glycoproteins that are secreted by specific cells of the immune system; they regulate inflammation and red blood cell production. They have an effect on the interactions between cells, and the behaviour of 
cells. The cytokines includes the interleukins, lymphokines and cell signal molecules, such as tumour necrosis factor, and the interferons, which trigger inflammation and infection responses, and possible links to cognitive ability. (www.sinobiological.com)

Insulin-like growth factor (IGF) is a peptide hormone that functions primarily to stimulate growth, but that also possesses some ability to decrease blood glucose levels, having insulin-like actions in some tissues, although far less potent than insulin in decreasing blood glucose concentrations. (www. britannica.com/science)

Brain derived neurotrophic factor (BDNF) is a protein produced inside nerve cells. It is the most prevalent growth factor in the central nervous system (CNS). It is essential for the development of the CNS and for neuronal plasticity (Autry and Monteggia, 2012)

Cortisol is one of the steroid hormones and is made in the adrenal glands. It is often called the "stress hormone" because of its connection to the stress response; however, because most bodily cells have cortisol receptors, it affects many different functions in the body. Cortisol can help control blood sugar levels, regulate metabolism, help reduce inflammation, and assist with memory formulation (www.hormone.org/hormones-and-health)

Beta amyloid is derived from an amyloid precursor protein and is thought to be the primary component of plaque characteristic in Alzheimer's disease. It is also referred to as A-beta, amyloid beta, amyloid beta peptide, and amyloid beta protein (www.merriawebster.com)

Acetoacetate is associated with energy metabolism. It is produced in the liver and is released into the bloodstream as an energy source during periods of fasting, exercise, or as a result of type 1 diabetes mellitus. Heart muscle and renal cortex respond more to acetoacetate than to glucose. The brain uses acetoacetate when glucose levels are low due to fasting or diabetes (www.medical-dictionary.com)

C-reactive protein (CRP) is a substance produced by the liver in response to inflammation.

A high level of CRP in the blood is a marker of inflammation. High CRP levels can indicate inflammation in the arteries of the heart, or risk of a heart attack. However, the CRP test is an extremely nonspecific test, and CRP levels can be elevated in any inflammatory condition. (www.healthline.com)

Irisin has been dubbed the "exercise hormone; irisin is produced when humans work up a sweat, and is thought to hold promise as weight-loss treatment, with possible links to telomere lengthening and brain functioning (www.livescience.com).

Norepinephrine is also referend to as noradrenaline or noradrenalin, and is an organic chemical in the catecholamine family that functions in the brain and body as a hormone and neurotransmitter (www. britannica.com/science)

Homocysteine is an amino acid. Having elevated levels of homocysteine in the blood is associated with atherosclerosis and blood clots. It has been suggested that high levels of homocysteine are associated with poorer mental functioning, leading to investigations into the role of homocysteine in Alzheimer's disease (medical-dictionary.com).

\section{References}

Allard, J.S., Ntekim, O., Johnson, S.P., Ngwa, J.S., Bond, V. and Pinder, D. (2017), "APOE $\varepsilon 4$ impacts upregulation of brain-derived neurotrophic factor after a six-month stretch and aerobic exercise intervention in mild cognitively impaired elderly African Americans: a pilot study", Experimental Gerontology, Vol. 87, pp. 129-136

Alzheimer's Society (2015), Exercise and Physical Activity, Alzheimer's Society.

Amjad, I., Toor, H., Niazi, I.K., Pervaiz, S., Jochumsen, M., Shafique, M., Haavik, H. and Ahmed, T. (2019), "Xbox 360 kinect cognitive games improve slowness, complexity of EEG, and cognitive functions in subjects with mild cognitive impairment: a randomized control trial", Games for Health Journal, Vol. 8 No. 2, pp. 144-152. 
Anderson, J., Calvo, D., Glickman, E., Gunstad, J. and Spitznagel, M. (2017), "The moderating role of insulin-like growth factor 1 in the relationship between cognitive and aerobic endurance change", Journal of Geriatric Psychiatry and Neurology, Vol. 30 No. 2, pp. 84-89.

Autry, A.E. and Monteggia, L.M. (2012), "Brain-Derived neurotrophic factor and neuropsychiatric disorders", Pharmacological Reviews, Vol. 64 No. 2, pp. 238-258.

Baker, L.D., Frank, L.L., Foster-Schubert, K., Green, P.S., Wilkinson, C.W., McTiernan, A., Plymate, S.R., Fishel, M.A., Watson, G.S., Cholerton, B.A., Duncan, G.E., Mehta, P.D. and Craft, S. (2010), "Effects of aerobic exercise on mild cognitive impairment", Archives of Neurology, Vol. 67 No. 1, pp. 71-79.

Bartlo, P. and Klein, P.J. (2011), "Physical activity benefits and needs in adults with intellectual disabilities: systematic review of the literature", American Journal on Intellectual and Developmental Disabilities, Vol. 116 No. 3, pp. 220-232.

Bowes, A., Dawson, A., Greasley-Adams, C. and McCabe, L. (2016), "Design of residential environments for people with dementia and sight loss: a structured literature review", British Journal of Visual Impairment, Vol. 34 No. 3, pp. 225-237.

Bowes, A., Dawson, A., Jepson, R. and McCabe, L. (2013), "Physical activity for people with dementia: a scoping study", BMC Geriatrics, Vol. 13 No. 1, p. 129.

Britannica Dictionary (science division) (2019), "Insulin-like growth factor", available at: www.britannica. com/science, (accessed 30 July 2020).

Chirles, T.J., Reiter, K., Weiss, L.R., Alfini, A.J., Nielson, K.A. and Smith, J.C. (2017), "Exercise training and functional connectivity changes in mild cognitive impairment and healthy elders", Journal of Alzheimer's Disease, Vol. 57 No. 3, pp. 845-856.

Chupel, M.U., Direito, F., Furtado, G.E., Minuzzi, L.G., Pedrosa, F.M., Colado, J.C., Ferreira, J.P., Filaire, E. and Teixeira, A.M. (2017), "Strength training decreases inflammation and increases cognition and physical fitness in older women with cognitive impairment", Frontiers in Physiology, Vol. 8, pp. 10-23.

Colcombe, S. and Kramer, A.F. (2003), "Fitness effects on the cognitive function of older adults: a Metaanalytic study", Psychological Science, Vol. 14 No. 2, pp. 125-130.

Csernansky, J.G., Dong, H. and Fagan, A.M. (2006), "Plasma cortisol and progression of dementia in subjects with alzheimer-type dementia", American Journal of Psychiatry, Vol. 163 No. 12, pp. 2164-2169.

Damirchi, A., Hosseini, F. and Babae, P. (2018), "Mental training enhances cognitive function and BDNF more than either physical or combined training in elderly women with mci: a small-scale study", American Journal of Alzheimer's Disease \& Other Dementiasr, Vol. 33 No. 1, pp. 20-29.

Dawson, A., Bowes, A., Kelly, F., Velzke, K. and Ward, R. (2015), "Evidence of what works to support and sustain care at home for people with dementia: a literature review with a systematic approach", BMC Geriatrics, Vol. 15 No. 1, pp. 59.

Douglas, S., James, I. and Ballard, C. (2004), "Non-pharmacological interventions in dementia", Advances in Psychiatric Treatment, Vol. 10 No. 3, pp. 171-179.

Economic and Social Research Council (2019), "Research with potentially vulnerable people", www.esrc. ukri.org, (accessed 1 June 2020).

El-Kader, S.M.A. and Al-Jiffri, O.H. (2016), "Aerobic exercise improves quality of life, psychological wellbeing and systemic inflammation in subjects with alzheimer's disease", African Health Sciences, Vol. 16 No. 4, pp. 1045-1055.

Gov, U.K. (2018), "Introduction to logic models", available at: www.gov.uk, (accessed 03 July 20).

Haeger, A., Costa, A.S., Schulz, J.B. and Reetz, K. (2019), "Cerebral changes improved by physical activity during cognitive decline: a systematic review on MRI studies", Neuroimage: Clinical, Vol. 23, p. 101933

Ho, R.T., Fong, T., Chan, W., Kwan, J., Chiu, P., Yau, J. and Lam, L. (2018), "Psychophysiological effects of dance movement therapy and physical exercise on older adults with mild dementia: a randomized controlled trial", Journals of Gerontology, Vol. 75 No. 3, pp. 560-570.

Hong, S.-G., Kim, J.-H. and Jun, T.-W. (2017), "Effects of 12-week resistance exercise on electroencephalogram patterns and cognitive function in the elderly with mild cognitive impairment: a randomized controlled trial", Clinical Journal of Sport Medicine, Vol. 28 No. 6, pp. 500-508.

Hormone.org (2019), available at: www.hormone.org/hormones-and-health. (accessed 30 July 2020). 
Hsu, C.L., Best, J.R., Davis, J.C., Nagamatsu, L.S., Wang, S., Boyd, L.A., Hsiung, G.R., Voss, M.W., Eng, J.J. and Liu-Ambrose, T. (2017), "Aerobic exercise promotes executive functions and impacts functional neural activity among older adults with vascular cognitive impairment", British Journal of Sports Medicine, Vol. 52 No. 3, pp. 184-191.

Junge, T., Ahler, J., Knudsen, H.K. and Kristensen, H.K. (2018), "The effect and importance of physical activity on behavioural and psychological symptoms in people with dementia: a systematic mixed studies review", Dementia, Vol. 0 No. 0, pp. e1-14.

Kim, J., Heo, J. and Kim, J. (2014), "The benefits of in-group contact through physical activity involvement for health and well-being among korean immigrants", International Journal of Qualitative Studies on Health and Well-Being, Vol. 9 No. 1, pp. 23517.

Kim, K.-H. and Lee, H.-B. (2018), "The effects of whole body vibration exercise intervention on electroencephalogram activation and cognitive function in women with senile dementia", Journal of Exercise Rehabilitation, Vol. 14 No. 4, pp. 586-591.

Kohanpour, M.-A., Peeri, M. and Azarbayjani, M.-A. (2017), "The effects of aerobic exercise with lavender essence use on cognitive state and serum brain-derived neurotrophic factor levels in elderly with mild cognitive impairment", Journal of Herbmed Pharmacology, Vol. 6 No. 2, pp. 80-84.

Lewis, K., Livsey, L., Naughton, R.J. and Burton, K. (2020), "Exercise and dementia: what should we be recommending?", Quality in Ageing and Older Adults, Vol. 21 No. 2, pp. 109-127.

Liu, I.T., Lee, W.J., Lin, S.Y., Chang, S.T., Kao, C.L. and Cheng, Y.Y. (2020), "Therapeutic effects of exercise training on elderly patients with dementia: a randomized controlled trial", Archives of Physical Medicine and Rehabilitation, Vol. 101 No. 5, pp. 762-769.

Mechling, H. (2008), "Dementia and physical activity", European Review of Aging and Physical Activity, Vol. 5 No. 1, pp. 33-36.

Medical Dictionary (2019), "Mechanism of action", available at: www.medical-dictionary. thefreedictionary.com (accessed 1 July 2020).

Merom, D., Grunseit, A., Eramudugolla, R., Jefferis, B., McNeill, J. and Anstey, K.J. (2016), "Cognitive benefits of social dancing and walking in old age: the dancing mind randomized controlled trial", Frontiers in Aging Neuroscience, Vol. 8, pp. 26-31.

Moher, D., Liberati, A., Tetzlaff, J. and Altman, D.G, The PRISMA Group (2009), "Preferred reporting items for systematic reviews and Meta-analyses: the PRISMA statement”, PLoS Medicine, Vol. 6 No. 7 , pp. e1000097.

Moore, G., Audrey, S., Barker, M., Bond, M., Bonell, C., Hardeman, W., Moore, L., O'Cathain, A., Tinati, T., Wight, D. and Bair, J. (2014), Process Evaluation of Complex Interventions: Medical Research Council Guidance. London, MRC Population Health Science Research Network.

Morris, J.K., Vidoni, E.D., Johnson, D.K., Van Sciver, A., Mahnken, J.D., Honea, R.A., Wilkins, H.M., Brooks, W.M., Billinger, S.A., Swerdlow, R.H. and Burns, J.M. (2017), "Aerobic exercise for alzheimer's disease: a randomized controlled pilot trial”, Plos One, Vol. 12 No. 2, pp. e1-14.

National Institute for Heath and Care Excellence (NICE) (2018), "Physical activity and the environment", NICE guideline (NG90), March 2018, London.

Otte, C., Hart, S., Neylan, T.C., Mamar, C.R., Yaffe, K. and Mohr, D.C. (2005), "A Meta-analysis of cortisol response to challenge in human aging: importance of gender", Psychoneuroendocrinology, Vol. 30 No. 1, pp. 80-91.

Pan, X. and Chee, K.H. (2019), "The power of weak ties in preserving cognitive function: a longitudinal study of older chinese adults", Aging and Mental Health, April 2019, 1-8.

Pedroso, R.V., Cancela, J.M., Ayán, C., Stein, A.M., Fuzaro, G., Costa, J.L.R., Fraga, F.J. and SantosGalduróz, R.F. (2018), "Effects of physical exercise on the P300 of elderly with alzheimer's disease", Journal of Physical Activity and Health, Vol. 15 No. 6, pp. 403-410.

Pluye, P. Robers, E. Cargo, M. Bartlett, G. ÒCathain, A. Griffiths, F. Boardman, F. Gagnon, M.P. Rousseau, M. and Robert, E. (2011), "Proposal: a mixed methods appraisal tool for systematic mixed studies reviews", available at: http://mixedmethodsappraisaltoolpublic.pbworks.com (accessed 20 May 2020).

Reiner, M., Niermann, C., Jekauc, D. and Woll, A. (2013), "Long-term health benefits of physical activitya systematic review of longitudinal studies", BMC Public Health, Vol. 13 No. 1, pp. 813-822. 
Rutherford, A., Bu, F., Dawson, A. and McCall, V. (2019), Literature Review to Inform the Development of Scotland's Volunteering Outcomes Framework, Scottish Government, Edinburgh.

Segal, S.K., Cotman, C.W. and Cahill, L.F. (2012), "Exercise-induced noradrenergic activation enhances memory consolidation in both normal aging and patients with amnestic mild cognitive impairment", Journal of Alzheimer's Disease, Vol. 32 No. 4, pp. 1011-1018.

Smith, J.C., Nielson, K.A., Antuono, P. and Lyons, J.-A. (2013), "Semantic memory functional MRI and cognitive function after exercise intervention in mild cognitive impairment", Journal of Alzheimer's Disease, Vol. 37 No. 1, pp. 197-215.

ten Brinke, L.F., Bolandzadeh, N., Nagamatsu, L.S., Hsu, C.L., Davis, J.C., Miran-Khan, K. and Liu-Ambrose, T. (2015), "Aerobic exercise increases hippocampal volume in older women with probable mild cognitive impairment: a 6-month randomised controlled trial", British Journal of Sports Medicine, Vol. 49 No. 4, pp. 248-254.

Tsai, C.L., Pai, M.C., Ukropec, J. and Ukropcová, B. (2019), "Distinctive effects of aerobic and resistance exercise modes on neurocognitive and biochemical changes in individuals with mild cognitive impairment. Current alzheimer's", Current Alzheimer Research, Vol. 16 No. 4, pp. 316-332.

Tsai, C.L., Ukropec, J., Ukropcova, B. and Pai, M.-C. (2018), "An acute bout of aerobic or strength exercise specifically modifies circulating exerkine levels and neurocognitive functions in elderly individuals with mild cognitive impairment”, Neurolmage: Clinical, Vol. 17, pp. 272-284.

van der Kleij, L.A., Petersen, E.T., Siebner, H.R., Hendrikse, J., Frederiksen, K.S., Sobol, N.A., Hasselbalch, S.G. and Garde, E. (2018), "The effect of physical exercise on cerebral blood flow in alzheimer's disease", Neurolmage: Clinical, Vol. 20, pp. 650-654.

Vardy, E.R., Rice, P.J., Bowie, P.C., Holmes, J.D., Grant, P.J. and Hooper, N.M. (2007), "Increased circulating insulin-like growth factor-I in late-onset alzheimer's disease", Journal of Alzheimer's Disease, Vol. 12 No. 4, pp. 285-290.

Vital, T.M., Soleman Hernandez, S., Stein, A.M., Garuffi, M. and Teixeira, C.V.L. (2016), "Resistance training, lipid profile, and homocysteine in patients with alzheimer's disease", International Journal of Gerontology, Vol. 10 No. 1, pp. 28-32.

World Health Organisation (2019), The Epidemiology and Impact of Dementia. Current State and Future Trends, WHO, Geneva.

Yerokhin, V., Anderson-Hanley, C., Hogan, M.J., Dunnam, M., Huber, D., Osborne, S. and Shulan, M. (2012), "Neuropsychological and neurophysiological effects of strengthening exercise for early dementia: a pilot study", Aging, Neuropsychology, and Cognition, Vol. 19 No. 3, pp. 380-401.

\section{Further reading}

Castellano, C.A., Paquet, N., Dionne, I.J., Imbeault, H., Langlois, F., Croteau, E., Tremblay, S., Fortier, M., Matte, J.J., Lacombe, G., Fülöp, T., Bocti, C. and Cunnane, S.C. (2017), "A 3-Month aerobic training program improves brain energy metabolism in mild alzheimer's disease: preliminary results from a neuroimaging study", Journal of Alzheimer's Disease, Vol. 56 No. 4, pp. 1459-1468.

NHS (2019), "Can dementia be prevented?", available at: www.nhs.uk (accessed 3 July 2020).

Rektorova, I., Klobusiakova, P., Balazova, Z., Kropacova, S., Sejnoha Minsterova, A. and Grmela, R. (2020), "Brain structure changes in nondemented seniors after six-month dance-exercise intervention", Acta Neurologica Scandinavica, Vol. 141 No. 1, pp. 90-97.

Silva, V.C., Ramos, F.J. and Freijas, E.M. (2007), "Alzheimer's disease in brazilian elderly has a relation with homocysteine but not with MTHFR polymorphisms", Arquivos de Neuro-Psiquiatria, Vol. 64 No. 4, pp. 941-945.

\section{About the authors}

Jan Pringle has worked as a systematic reviewer and researcher at the Scottish Collaboration for Public Health Research and Policy for the past 6 years; she has an interest in all interventions that promote health and well-being. Jan Pringle is the corresponding author and can be contacted at: jpringl2@exseed.ed.ac.uk

Ruth Jepson is a Professor of Public Health in Social Science. She has expertise in a range of systematic review methods, and leads on numerous public health research projects. 
Alison Dawson is a Senior Research Fellow at the Faculty of Social Sciences, University of Stirling, and co-leader of the Faculty's Dementia and Ageing Research Group. Her current research interests include the role of technology in providing support services for older people, time use in unpaid care/support, promoting movement and activity in care homes, and the role of building design in supporting people with dementia.

Louise McCabe is a Senior Lecturer in Dementia Studies, University of Stirling, with expertise in qualitative research that engages with people with dementia and their families and focuses on the role and impact of care services.

Alison Bowes is a Professor in Sociology and Dean of the Faculty of Social Sciences, University of Stirling. She researches issues relating to supporting better ageing and better living with dementia, and has published widely in this area.

For instructions on how to order reprints of this article, please visit our website: www.emeraldgrouppublishing.com/licensing/reprints.htm

Or contact us for further details: permissions@emeraldinsight.com 